\title{
The Effect of Embryo Transfer Process Duration on Implantation Success
}

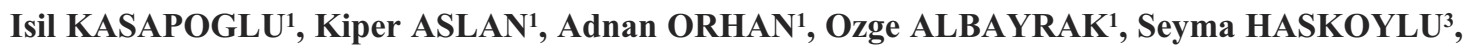 \\ Goktan KUSPINAR ${ }^{2}$, Berrin AVCI², Gurkan UNCU' ${ }^{1}$
}

Bursa, Türkiye

ABSTRACT

OBJECTIVE: To evaluate the effect of the embryo transfer duration of standard and simple embryo transfer method.

STUDY DESIGN: This study was a retrospective cohort study conducted at a tertiary ART Centre, between June 2018- September 2018. Day 5 fresh embryo transferred patients aged between 18 - 40, BMI $<35 \mathrm{~kg} / \mathrm{m}^{2}$ without uterine pathology were enrolled in the study. Patients were divided into two groups. Group-1 consisted of patients who had successful implantation and Group-2 consisted of patients who did not have implantation. Groups were compared according to their embryo transfer durations. Ninetytwo patients were enrolled in the study. Also, sub-steps of as; cleaning of the cervical mucus and placing the outer catheter in the cervix, loading the embryo to the catheter, the period between embryo loading and embryo transfer, and following that, time spent for retracting the outer catheter evaluated.

RESULTS: Between Group-1 and Group-2, there was no significant difference for the period of cervical cleaning and placing the outer catheter into the cervix (Respectively; $63 \mathrm{sec}$ vs. $76 \mathrm{sec} ; p=0.18$ ), the period of embryo loading (Respectively; $69 \mathrm{sec}$ vs. $71 \mathrm{sec} ; p=0.46$ ), the period between embryo loading and embryo transfer (Respectively; 10 sec vs. $10 \mathrm{sec;} p=0.74$, retracing the outer catheter (Respectively; $25.5 \mathrm{sec}$ vs. $24 \mathrm{sec} ; p=0.42$ and the total period of embryo transfer (182sec vs. $182.5 \mathrm{sec} ; p=0.55)$.

CONCLUSION: The embryo transfer duration is not related to implantation rates. The duration of the embryo transfer process steps is not a distinguishing factor if a good-quality embryo transfer is done.

Keywords: Embryo transfer, Embryo transfer duration, Implantation, In-vitro fertilization

Gynecol Obstet Reprod Med 2022;28(2):151-156

\section{Introduction}

Embryo transfer (ET) is one of the latest and most crucial steps which determines the success of in vitro fertilization

${ }^{1}$ Department of Obstetrics and Gynecology, Uludag University School of Medicine, Bursa, Türkiye

2 Department of Histology and Embryology, Uludag University School of Medicine, Bursa, Türkiye

${ }^{3}$ Uludag University School of Medicine, Bursa, Türkiye

Address of Correspondence: Kiper Aslan

Uludag Universitesi Tip Fakultesi Izmir

yolu 17. km, Gorukle, Bursa, Türkiye

kiperaslan@yahoo.com.tr

Submitted for Publication: 22.03.2020 Revised for Publication: 15.09.2020

Accepted for Publication: 27.01.2021 Online Published: 30.03.2021

ORCID IDs of the authors: IK: 0000-0002-1953-2475

KA: 0000-0002-9277-7735, AO: 0000-0002-7558-8166

ÖA: 0000-0001-7798-8799, SुH: 0000-0002-6697-8533

GK: 0000-0002-0338-8368, BA: 0000-0001-8135-5468 GU: 0000-0001-7660-8344

\begin{tabular}{c|c}
\hline Quick Response Code: & Access this article online \\
\cline { 2 - 2 } & $\begin{array}{c}\text { Website: www.gorm.com.tr } \\
\text { e- mail: info@gorm.com.tr }\end{array}$ \\
\cline { 2 - 2 } & DOI:10.21613/GORM.2021.1156 \\
\hline
\end{tabular}

How to cite this article: Kasapoglu I. Aslan K. Orhan A. Albayrak O. Haskoylu S. Kuspinar G. Avcı B. Uncu G. The Effect of Embryo Transfer Process Duration on Implantation Success. Gynecol Obstet Reprod Med. 2022;28(2):151-156
(IVF)-intracytoplasmic sperm injection (ICSI) cycles. In recent years, $80 \%$ of patients can reach the ET phase with the help of the progress of embryology laboratories and the ovarian stimulation protocols, aiming to enhance the success of IVF-ICSI cycles (1). Thus, clinical success rates do not approach these rates after the transfer. However, for most of the transferred embryos, the implantation failures are because of the uterine receptivity, and $30 \%$ of them are associated with the ET technique $(1,2)$.

Besides endometrial receptivity and the embryo's quality, optimal standardization of the ET technique is not possible because the technique's success is mainly operator-dependent (3). Even so, modifications are suggested to improve the technique. These are: transferring accompanied by ultrasonography and observing the catheter throughout the process (4), minimizing the endometrial trauma (5), using a soft catheter (6), and minimizing the ET duration (7).

There is a common enough view that embryo transfer should be done with an atraumatic method to minimize uterine contractility (8). Nevertheless, there is insufficient proof in the literature about the ET duration. Research studies indicate that total embryo transfer duration should be shortened, even 
that it should be less than 120 seconds; otherwise, it will negatively affect the clinical success (7). It is suggested that the ET duration should be shortened, and the most important reason for this is reducing the embryo's exposure to the adverse effects of exterior heat and light as much as possible after loading the embryo into the catheter (7). Thus, the process may be extended to reduce endometrial trauma. Most of the research about ET duration is based on the time between drawing the embryo into the catheter and placing it into the endometrial cavity. Most of the studies include transfers, which are time-consuming and robust in the analysis (7). Challenging ET processes affect ET duration negatively. In addition to this, there will be adverse effects, which will decrease the success of the process such as catheter contamination by blood, and if cervical manipulation is necessary it will trigger uterine contractility (9).

This study aimed to evaluate the effect of the duration of the standard and simple ET method utilized on clinical success so that the effect of ET duration on implantation was evaluated without including difficult ET processes. Rather than considering only total ET duration, the time spent on each sub-step throughout the entire process, such as cleaning of the cervical mucus and placing the outer catheter into the cervix, loading the embryo into the catheter, loading, and transfer of the embryo, and retracting the outer catheter was evaluated separately.

\section{Material and Method}

This retrospective study was conducted at a Tertiary University Hospital IVF Centre between June 2018 and September 2018, with 92 patients introduced to the ICSI cycle and on whom fresh ET was performed on the fifth day with various indications. Uludag University Institutional Review Board approved the study protocol with the medical ethics committee number $2019-1 / 24$ on 15.01.2019. The study was conducted in accordance with the declaration of Helsinki.

A digital timer measured ET periods, and the prospectively recorded data were evaluated retrospectively. Ninetytwo patients were separated into two subgroups, those who achieved implantation after ET (Group-1, n=56) and did not achieve implantation (Group-2, n=36).

The criteria for these patients' involvement were: being at an age between 18 and $40, \mathrm{BMI}<35 \mathrm{~kg} / \mathrm{m}^{2}$, being at a stage where there was no diagnosed uterine pathology, being transferred with one good-quality fresh blastocyst on the transfer day (fifth day) after controlled ovarian hyperstimulation, and needing neither cervical manipulation nor catheter guidewire. Patients whose ET was simply done were also included in the study. Excluded cases were: BMI $>35 \mathrm{~kg} / \mathrm{m} 2$, frozen ET cycles, diagnosed with uterine pathologies, previous endometrial cavity surgeries, difficult ET (necessity to use catheter guide or tenaculum during the transfer), presence of blood or em- bryo retention in the catheter after the transfer and requirement of reloading or change of the catheter.

Controlled ovarian hyperstimulation cycles were evaluated according to all of the patients' ovarian reserve and BMI, and implemented by the antagonist protocol of 150-450 IU daily doses of gonadotropin. Starting from the fifth day of the stimulation, we monitored intensively by serum estradiol and progesterone levels and serial transvaginal ultrasonographic measurements. According to transvaginal ultrasonography, when the dominant follicle reached the size of $14 \mathrm{~mm}$, subcutaneous Cetrorelix 0.25/day was applied as an antagonist (flexible antagonist protocol). Oocyte maturation was triggered by 250 micrograms of subcutaneous recombinant choriogonadotropin alfa (rHCG) (Ovitrelle; Merck Serono) when at least two follicles that had reached the size of $18 \mathrm{~mm}$ or three follicles that had reached the size of $17 \mathrm{~mm}$ were identified by ultrasonography. If the serum progesterone level was above $1.5 \mathrm{ng} / \mathrm{ml}$ on the day on which hCG was implemented, instead of going through the embryo transfer process, embryos were frozen, and frozen embryo transfer was performed on another menstrual cycle. Oocyte pick-up (OPU) was done 34-36 hours after rhCG implementation under general anesthesia accompanied by transvaginal ultrasonography.

Intracytoplasmic sperm injection was applied to all oocytes. Embryo transfers were done on the fifth day, with good-quality (10) embryos (concordance with its day, blastocoel expansion rate between 1-4, embryos at blastocyst stage whose inner and outer cell masses were A or B quality), by using a soft catheter (Cook Medical) and the standard amount of culture medium. All embryology process was done by the same embryologist.

All the transfers were performed with soft catheters by one experienced clinician from the research team with abdominal ultrasonography guidance when the patients' bladders were full. The preparation phase of embryo transfer was conducted in the same way for all the patients. The patients were examined in the dorsolithotomy position, and after their cervical examinations were done, they were prepared for the transfer by cleaning of the cervical mucus and secretions with physiological serum. Following the cleaning of the cervical mucus, the outer catheter was placed and positioned. The inner catheter's position was monitored by abdominal ultrasonography, and ET was performed one $\mathrm{cm}$ below the fundus.

An embryologist examined the inner catheter for retention, blood, and mucus contamination after the transfer. Following this, the outer catheter was pulled out without waiting. All of the patients were rested for an hour after the transfer.

For luteal phase support, vaginal progesterone $2 \times 1$ (Crinone gel 8\%; Merck Serono, Switzerland) was used starting on the OPU day. Serum Beta-subunit of human chorionic gonadotropin ( $\beta$-hCG) examination was done 12 days after the 
embryo transfer. Luteal support was stopped if $\beta-\mathrm{hCG}$ was negative; if positive, the support treatment was continued until the 8 th gestational week. Implantation diagnosis was made by identifying the gestational sac by ultrasonography.

Groups that had successful implantations and those who did not (Group-1 and Group-2) were compared according to their ET duration and total period (second-second) difference. Secondary results were the sub-steps of the ET transfer: the period for cleaning of the cervical mucus and placing the outer catheter in the cervix, the period of loading the embryo into the catheter, the period between embryo loading and embryo transfer, and following that, the time spent for retracting the outer catheter. Each step was recorded by a digital timer and evaluated separately.

The patient gave written informed consent for an Institutional Review Board approved study protocol.

Statistical analysis of the data was calculated with the IBM SPSS Statistics (Statistical Package for the Social Sciences) version 23.0 (IBM Corporation, Armonk, NY, the USA). The Shapiro-Wilk test was used to determine if the data had a normal distribution curve or not. Illustrative statistics were indicated as mean \pm SD and median $(25 \%$ and $\% 75 \%$ percentile values). The Mann-Whitney U test was used to compare the groups, which were not distributed normally. The significance level was identified as $p \leq 0.05$.

\section{Results}

The time durations evaluated are shown in table I. The clinic and laboratory characteristics of Group-1 and Group-2 are given in table II. The patients' average age, BMI, and serum AMH levels did not significantly differ between the groups ( $p=0.32, p=0.06, p=0.33$, respectively). No statistically significant difference was found between the groups regarding basal FSH, LH, or estradiol levels ( $p=0.14, \mathrm{p}=0.93, p=0.06$, respectively). The total amount of aspirated oocytes, mature oocytes, and fertilization rates did not show a statistical difference between the groups $(p=0.06, p=0.06$, and $p=0.5$, respectively) (Table II). Serum estradiol levels that were estimated on the day on which hCG was implemented were similar between groups. $(p=0.06)$ (Table II).

\section{Table I: Time intervals evaluated}

- The period of cervical cleaning and placing the outer catheter (sec)

- The period of loading the embryo into the inner catheter (sec)

- The period between embryo loading and embryo transfer (sec)

- The period of retracting the outer catheter after the embryo transfer (sec)

- The period of total embryo transfer (sec)

Since only the cases on which one embryo transfer was performed were included in the research, transferred embryo amounts did not show a statistically significant difference between the groups.

There were no significant differences between Group-1 and Group-2, concerning the period of cervical cleaning and

Table II: Characteristics of groups

\begin{tabular}{|c|c|c|c|}
\hline & $\begin{array}{c}\text { Group-1 } \\
\text { (Implantation negative) } \\
{[\mathrm{n}=56]}\end{array}$ & $\begin{array}{c}\text { Group-2 } \\
\text { (Implantation positive) } \\
{[n=36]}\end{array}$ & $p$ \\
\hline Age (year) & $32.6+4.3$ & $31.8+4.4$ & .32 \\
\hline BMI (kg/m2) & $25.5+4.4$ & $27.4+4.7$ & .06 \\
\hline Infertility period (year) & $6.8+4.7$ & $6.9+4.4$ & .73 \\
\hline Infertility Etiology & & & .44 \\
\hline Unexplained & $13(23.2 \%)$ & $9(25 \%)$ & \\
\hline Male Factor & $13(23.2 \%)$ & $9(25 \%)$ & \\
\hline DOR & $22(39.3 \%)$ & $13(36 \%)$ & \\
\hline Tubal Factor & $6(10.7 \%)$ & $3(8.3 \%)$ & \\
\hline Anovulation & $2(3.5 \%)$ & $2(5.5 \%)$ & \\
\hline Basal FSH & $5.7+1.8$ & $5.5+3.7$ & .14 \\
\hline Basal LH & $4.2+2.8$ & $3.9+2.1$ & .93 \\
\hline Basal E2 & $51.1+6.4$ & $38.3+3.5$ & .06 \\
\hline $\mathrm{AMH}$ & $2.9+0.3$ & $3.2+0.3$ & .33 \\
\hline hCG day E2 & $1652.8+196.1$ & $2204.8+236.3$ & .06 \\
\hline Total oocyte count & $11.3+7.1$ & $14+6.4$ & .06 \\
\hline MII oocyte count & $8.9+5.9$ & $11+5.1$ & .06 \\
\hline Fertilization rate & $.53+.22$ & $.62+.17$ & .50 \\
\hline
\end{tabular}


placing the outer catheter into the cervix (Respectively; $63 \mathrm{sec}$ vs. $76 \mathrm{sec} ; p=0.18$ ), the period of embryo loading (Respectively; $69 \mathrm{sec}$ vs. $71 \mathrm{sec} ; p=0.46$ ), the period between embryo loading and embryo transfer (Respectively; $10 \mathrm{sec}$ vs. 10sec; $p=0.74$ ), the period of retracting the outer catheter (Respectively; $25.5 \mathrm{sec}$ vs. $24 \mathrm{sec} ;=0.42$ ) and the total period of the loading (Respectively; $182 \mathrm{sec}$ vs. $182.5 \mathrm{sec} ; p=0.55$ ) (Table III). The duration of the total embryo transfer procedure was shown for two groups (Figure 1).

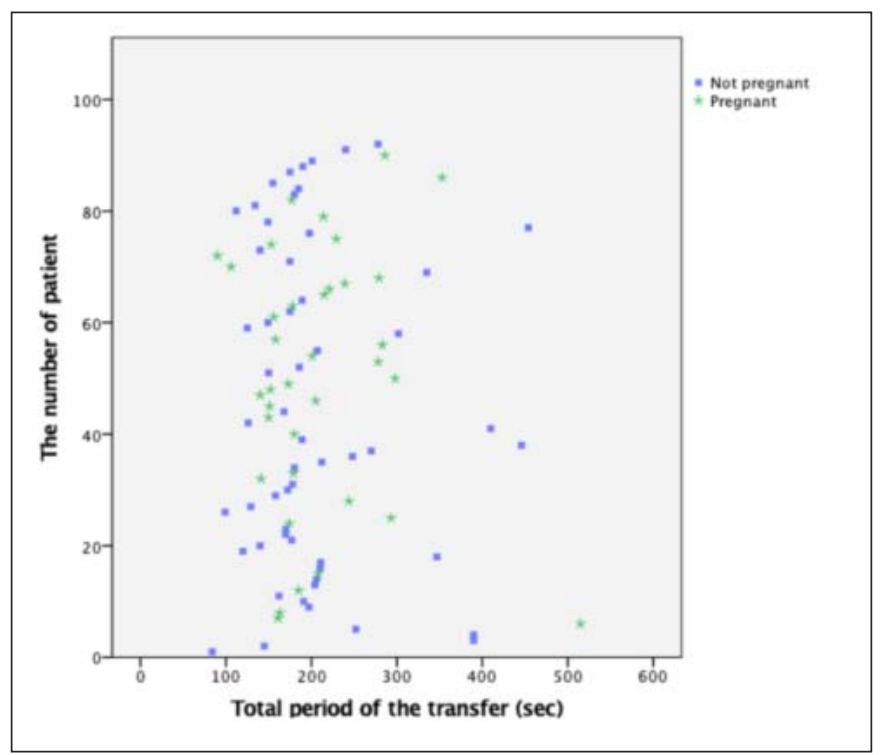

Figure 1: Duration of the total embryo transfer procedure

\section{Discussion}

In this research, we have found that ET duration of ICSI cycles that had standardized embryo quality and luteal phase support did not show significant differences between patients whose implantation results were positive and negative. The periods of the different sub-steps of the ET process were not influential in implantation success. The patients transferred with one good-quality embryo on the fifth day, utilized as an easy ET transfer, and did not have a uterine pathology examined to evaluate implantation separately from embryo quality.

Embryo transfer is the last step of the IVF-ICSI cycles and has critical importance on clinical success. Globally, longer transfer duration was thought to be a sign of a difficult trans- fer period. However, currently, novel studies focused on the determination of other markers to define difficult transfer (11). It is emphasized that some of the embryo transfer modifications may positively affect clinical results (8). Proven modifications are: doing the transfer with the guidance of ultrasonography to observe the transfer catheter possible during the entire process (4), reducing the endometrial trauma to a minimum (5), and using a soft catheter (6). Although studies indicate that minimizing the total duration of the ET process may affect the clinical success (7), there is not enough proof to agree on the strict idea that shortening the ET process's total period will enhance clinical success. It is propounded that when good-quality embryos are transferred, the clinical success rate will not decrease despite the extended ET period (12). Also, the recent guideline on embryo transfer of the American Society for Reproductive Medicine did not focus on any cut-off values for the duration of the ET (13). For this purpose, besides the total ET period, the periods of each sub-step of the ET processes were evaluated separately for their effects on clinical success.

During the embryo transfer, the suggested necessary precautions were doing the transfer without any trauma in the endometrial cavity and without triggering any uterine contraction (8). It is known that uterine contractions, triggered during the embryo transfer, will affect the results negatively. Some of the factors that caused uterine contractility are: clasping the cervix with a tenaculum (14), allowing the inner catheter to reach the uterine fundus (15), and using a guidewire to pass the cervix (16), which are also factors of a challenging transfer. Therefore, in our research, especially challenging transfer cases were not considered because the negative factors listed above may also have adverse effects on the results apart from the extended ET duration. Most of the studies about ET duration and period do consider challenging transfers additionally. A prospectively conducted research study showed that even if challenging transfer cases were involved, ET speed would not affect clinical results as long as good-quality embryo transfer was done. This result is similar to our research.

Some studies about embryo transfer duration emphasized the necessity to complete the transfer as soon as possible (7). It is mentioned that transfers, which take longer than $120 \mathrm{sec}-$ onds, are correlated with adverse clinical results (7). Even if

Table III: Time intervals of the embryo transfer procedure (sec) for the groups

\begin{tabular}{|c|c|c|c|}
\hline & $\begin{array}{c}\text { Group-1 } \\
\text { (Implantation negative) } \\
{[\mathrm{n}=56]}\end{array}$ & $\begin{array}{c}\text { Grup-2 } \\
\text { (Implantation positive) } \\
{[\mathrm{n}=36]}\end{array}$ & $p$ \\
\hline The period of cervical cleaning and placing the outer catheter & $63(43.5-95.5)$ & $76(48.5-116.5)$ & .18 \\
\hline The period of loading the embryo into the inner catheter & $69(55-90.5)$ & $71(49.5-77)$ & .46 \\
\hline The period between embryo loading and embryo transfer & $10(6-16)$ & $10(6.5-16)$ & .74 \\
\hline The period of which the outer catheter stays inside after the embryo transfer & $25.5(20-31.5)$ & $24(20-30)$ & .42 \\
\hline The period of total embryo transfer & $182.0(152.5-211.5)$ & $182.5(157-241.5)$ & .55 \\
\hline
\end{tabular}


the ET duration exceeded 60 seconds significantly lower pregnancy rates have been reported (17).

However, these studies did not mention the operators who did the transfers. The success of the ET process success is also dependent on the operator, so if the research were conducted with more than one operator, it would not allow for revealing an objective assessment. In our study, all of the transfers were done by one operator.

Among the present research in the literature, most of the research about the ET period reveals the time between drawing the embryo into the catheter and the transfer as the total duration of the process. However, other steps of the ET process extend the transfer period as much as drawing the embryo into the catheter does. One of the fundamental purposes of our research was to compare the duration of the other substeps of the ET transfer as well.

The period after drawing the embryo into the catheter was considered to estimate the ET duration in a couple of studies $(7,18)$, emphasizing that transfer duration does not have a relation with clinical success rates after drawing the embryo into the catheter, which is similar to our results (18). A more recent study similarly concluded that longer ET duration does not negatively affect implantation, clinical pregnancy, or live birth rates (19). In our study, the difference is that for estimating the total period, we start with the cleaning of the cervical mucus and placing the speculum, which is the beginning of the transfer process. For enhancing the success of embryo transfer, it is shown that removing the cervical mucus before placing the catheter increases clinical success rates (8). During the cervix's cleaning, it is suggested not to cause any trauma or bleeding, and if the suggestions are considered, the transfer period may be extended. Nevertheless, no research evaluates the effect of this extended period on ET success.

Moreover, the effect of the extended period on uterine contraction remains unknown. No difference was found in the comparisons between the two groups about the period of cleaning the cervical mucus, which is considered in a study for the first time in our research. However, as stated, there is a need for research, which focuses on the effects on uterine contractility if the period of this step is extended.

There are limited numbers of studies with conflicting results about retracting the outer catheter, which may reduce uterine contractility if placed in the cervix slowly and carefully to make an atraumatic embryo transfer. According to Martinez et al., retracting the outer catheter right after ET or retracting it after a 30 -second wait does not produce different pregnancy results (20). Another retrospective study, which evaluates the retraction period of the outer catheter, has similar results (21). However, some studies emphasized that for the patients whose previous transfer was done without waiting for retracting the outer catheter, it may be beneficial to wait before pulling out the outer catheter. This research shows that the period of retracting the outer catheter after the transfer of the embryo into the cavity does not affect implantation success.

The major limitation of our study is the evaluation of the implantation rates for a particular and small cohort from a single institute and it could be discussed if these outcomes could be extrapolated to all ET cycles. To maximize and standardization of the outcomes, further prospective studies in large cohorts would be intriguing.

In conclusion, we have shown that the total ET duration is not related to implantation rates. The duration of the ET process steps is not a distinguishing factor if a good-quality embryo transfer is done.

Acknowledgment: None

Conflict of interest: All authors state that there is no conflict of interest.

Funding: None

Ethical approval: Uludag University Institutional Review Board approved the study protocol with the medical ethics committee number 2019-1/24 on 15.01.2019.

Authors' contribution: Data collection, statically analysis, writing: IK, KA, AO; Embryology laboratory Timer Settings: BA, GK, SH; Folliculometry, Oocyte pick up, Embryo Transfer: $I K, K A, G U$, Reviewing and revising the manuscript: $G U$.

\section{References}

1. Mansour RT, Aboulghar MA. Optimizing the embryo transfer technique. Hum Reprod. 2002;17(5):1149-53. Doi: 10.1093/humrep/17.5.1149.

2. Brown J, Buckingham K, Buckett W, Abou-Setta AM. Ultrasound versus "clinical touch" for catheter guidance during embryo transfer in women. Cochrane Database Syst Rev. 2016;3:CD006107. Doi: 10.1002/14651858. CD006107.pub4.

3. Karande VC, Morris R, Chapman C, Rinehart J, Gleicher $\mathrm{N}$. Impact of the "physician factor" on pregnancy rates in a large assisted reproductive technology program: do too many cooks spoil the broth? Fertil Steril. 1999; 71(6):1001-9. Doi: 10.1016/s0015-0282(99)00139-9.

4. Matorras R, Urquijo E, Mendoza R, Corcóstegui B, Expósito A, Rodríguez-Escudero FJ. Ultrasound-guided embryo transfer improves pregnancy rates and increases the frequency of easy transfers. Hum Reprod. 2002; 17(7): 1762-6. Doi: 10.1093/humrep/17.7.1762.

5. Mansour R, Aboulghar M, Serour G. Dummy embryo transfer: A technique that minimizes the problems of embryo transfer and improves the pregnancy rate in human in vitro fertilization. Fertil Steril. 1990;54(4):678-81. Doi: 10.1016/s0015-0282(16)53829-1.

6. Van Weering HGI, Schats R, McDonnell J, Vink JM, Vermeiden JPW, Hompes PGA. The impact of the embryo transfer catheter on the pregnancy rate in IVF. Hum 
Reprod. 2002 ;17(3):666-70. Doi: 10.1093/hum$\mathrm{rep} / 17.3 .666$

7. Matorras R, Mendoza R, Expósito A, Rodriguez-Escudero FJ. Influence of the time interval between embryo catheter loading and discharging on the success of IVF. Hum Reprod. 2004;19(9):2027-30.Doi: 10.1093/humrep/deh 366

8. Penzias A, Bendikson K, Butts S, Coutifaris C, Falcone T, Fossum G, et al. Performing the embryo transfer: a guideline. Fertil Steril. 2017;107(4):882-96 Doi: 10.1016/j. fertnstert.2017.01.025

9. Goudas VT, Hammitt DG, Damario MA, Session DR, Singh AP, Dumesic DA. Blood on the embryo transfer catheter is associated with decreased rates of embryo implantation and clinical pregnancy with the use of in vitro fertilization-embryo transfer. Fertil Steril. 1998;70:87882. Doi: 10.1016/s0015-0282(98)00315-x

10. Racowsky C, Vernon M, Mayer J, Ball GD, Behr B, Pomeroy KO, et al. Standardization of grading embryo morphology. Fertil Steril. 2010;94(3):1152-3. Doi: 10.1016/j.fertnstert.2010.05.042

11. Kava-Braverman A, Martinez F, Rodriguez I, Alvarez M, Barri PN, Coroleu B. What is a difficult transfer? Analysis of 7,714 embryo transfers: the impact of maneuvers during embryo transfers on pregnancy rate and a proposal of objective assessment. Fertil Steril. 2017;107:657-63.e1. Doi: 10.1016/j.fertnstert.2016.11.020

12. Ciray HN, Tosun S, Hacifazlioglu O, Mesut A, Bahceci M. Prolonged duration of transfer does not affect outcome in cycles with good embryo quality. Fertil Steril. 2007; 87(5):1218-21. Doi:10.1016/j.fertnstert. 2006.08.100

13. Practice Committee of the American Society for Reproductive Medicine. Performing the embryo transfer: a guideline. Fertil Steril 2017;107(4):882-96. Doi: 10.1016/ j.fertnstert.2017.01.025.
14. Dorn C, Reinsberg J, Schlebusch H, Prietl G, van der Ven H, Krebs D. Serum oxytocin concentration during embryo transfer procedure. Eur J Obstet Gynecol Reprod Biol. 1999;87(1):77-80. Doi: 10.1016/s0301-2115(99)00077-9.

15. Lesny P, Killick SR, Robinson J, Raven G, Maguiness SD. Junctional zone contractions and embryo transfer: Is it safe to use a tenaculum? Hum Reprod. 1999;14(9): 2367-70. Doi: 10.1093/humrep/14.9.2367.

16. Fraser IS. Prostaglandins, prostaglandin inhibitors and their roles in gynaecological disorders. Baillieres Clin Obstet Gynaecol. 1992;6(4):829-57. Doi: 10.1016/s09503552(05)80191-9.

17. Cetin MT, Kumtepe Y, Kiran H, Seydaoglu G. Factors affecting pregnancy in IVF: age and duration of embryo transfer. Reprod Biomed Online. 2010;20(3):380-6. Doi: 10.1016/j.rbmo.2009.12.008.

18. Nouri K, Tempfer CB, Walch K, Promberger R, Dag S, Ott J. Predictive value of the time interval between embryo loading and transfer for IVF/ICSI success: A prospective cohort study. Reprod Biol Endocrinol. 2015;13:51. Doi: 10.1186/s12958-015-0048-6.

19. Lee MS, Cardozo ER, Karmon AE, Wright DL, Toth TL. Impact of transfer time on pregnancy outcomes in frozenembryo transfer cycles. Fertil Steril. 2018;109(3):467472. Doi: 10.1016/j.fertnstert.2017.11.031.

20. Martínez F, Coroleu B, Parriego M, Carreras O, Belil I, Parera N, et al. Ultrasound-guided embryo transfer: Immediate withdrawal of the catheter versus a 30 second wait. Hum Reprod. 2001;16(5):871-4. Doi: 10.1093/humrep/16.5.871.

21. Sroga JM, Montville CP, Aubuchon M, Williams DB, Thomas MA. Effect of delayed versus immediate embryo transfer catheter removal on pregnancy outcomes during fresh cycles. Fertil Steril. 2010;93(6):2088-90. Doi: 10. 1016/j.fertnstert.2009.07.1664. 